$$
\varphi(M)=\frac{a_{1} / \delta_{1}+a_{2} / \delta_{2}+\cdots+a_{n} / \delta_{n}}{1 / \delta_{1}+1 / \delta_{2}+\cdots+1 / \delta_{n}},
$$

except when $M$ is in $H_{i}$; then $\varphi(M)=a_{i}(i=1,2,3, \cdots, n) .^{*}$

The function $\varphi(M)$ is continuous in space of $n$ dimensions. I wish to show that it is continuous in the space $S$. It is sufficient to show that the distance $\delta$ of a point $M$ from a closed set $H$ is a continuous function of $M$, vanishing on $H$.

Let $(A, B)$ denote the distance between two points $A, B$ of the metric space $S$. Then we have the following fundamental property of distance: If $A, B, C$ are any three points of $S$,

$$
(A, B) \leqq(A, C)+(C, B)+\dagger
$$

If $H$ is a closed set the distance $(M, X)$, where $X$ is in $H$, has a minimum $\delta$ at a point $X_{0}$. We call $\delta=\left(M, X_{0}\right)$ the distance from $M$ to $H$. Let $M^{\prime}$ be any other point of $H$, and $\delta^{\prime}=\left(M^{\prime}, X_{0}{ }^{\prime}\right)$ be the corresponding distance from $H$. Then we have

$$
\begin{aligned}
\delta=\left(M, X_{0}\right) \leqq\left(M, X_{0}{ }^{\prime}\right) & \leqq\left(M, M^{\prime}\right)+\left(M^{\prime}, X_{0}{ }^{\prime}\right) \\
& \leqq\left(M, M^{\prime}\right)+\delta^{\prime} .
\end{aligned}
$$

Similarly, $\delta^{\prime} \leqq \delta+\left(M, M^{\prime}\right)$. Therefore $\left|\delta-\delta^{\prime}\right| \leqq 2\left(M, M^{\prime}\right)$, and the continuity of $\delta$ is established.

UNIVERSITY OF IOWA, Iowa City, Iowa. February 28, 1920.

\title{
CERTAIN ITERATIVE CHARACTERISTICS OF BILINEAR OPERATIONS.
}

BY DR. NORBERT WIENER.

\section{Introduction.}

IN a recent paper $\ddagger$ the author has developed the necessary and sufficient condition that a bilinear operation in two variables should generate by iteration all rational operations with

* Vallée-Poussin, loc. cit., p. 118.

$\dagger$ Hausdorff, loc. cit., p. 211. See also Fréchet, "Les notions de limite et de distance," Transactions Amer. Math. Society, vol. 19 (1918), p. 54.

$\ddagger$ Annals of Mathematics, vol. 21, No. 3 (March, 1920), pp. 157-165. 
rational coefficients. This is a particular case of the general problem of determining just what operations any given bilinear operation will generate by iteration. While the complete solution of this problem is still to be accomplished, it is the purpose of this paper to develop methods of attack which will yield, in particular, an important necessary condition that each of two operations generate the other by iteration.

\section{Definitions.}

A bilinear operation is an operation $\varphi(x, y)$ of the form

$$
\frac{A_{\phi}+B_{\phi} x+C_{\phi} y+D_{\phi} x y}{E_{\phi}+F_{\phi} x+G_{\phi} y+H_{\phi} x y} \text {. }
$$

In this paper the coefficients will be supposed to be rational, $\infty$ will be considered a proper value and argument for a bilinear operation, and if $\varphi\left(x_{1}, y_{1}\right)$ is indeterminate as it stands, it shall be given the value $\lim _{x=x_{1}, y \doteq y_{1}} \varphi(x, y)$ if this is
determinate.

A bilinear operation will be said to be reduced if $A_{\phi}$, $B_{\phi}+C_{\phi}, D_{\phi}, E_{\phi}, F_{\phi}+G_{\phi}$, and $H_{\phi}$ form a relatively prime set of integers.

$\Delta_{\phi}$ is defined as

$$
\| \begin{array}{lr}
\left|\begin{array}{ll}
A_{\phi} & B_{\phi}+C_{\phi} \\
E_{\phi} & F_{\phi}+G_{\phi}
\end{array}\right|\left|\begin{array}{ll}
A_{\phi} & D_{\phi} \\
E_{\phi} & H_{\phi}
\end{array}\right| \\
\left|\begin{array}{ll}
A_{\phi} & D_{\phi} \\
E_{\phi} & H_{\phi}
\end{array}\right|\left|\begin{array}{ll}
B_{\phi}+C_{\phi} & D_{\phi} \\
F_{\phi}+G_{\phi} & H_{\phi}
\end{array}\right|
\end{array} \mid .
$$

The following definitions are made:

$$
\begin{aligned}
J_{\phi}(u, v) & =A_{\phi} v^{2}+\left(B_{\phi}+C_{\phi}\right) u v+D_{\phi} u^{2}, \\
K_{\phi}(u, v) & =E_{\phi} v^{2}+\left(F_{\phi}+G_{\phi}\right) u v+H_{\phi} u^{2}, \\
L_{\phi} & =v J_{\phi}-u K_{\phi} .
\end{aligned}
$$

We shall term the roots of $L_{\phi}(u, 1)=0, r_{1}(\varphi), r_{2}(\varphi), r_{3}(\varphi)$. We shall call a root of $L_{\phi}(u, 1)=0$ free if it is not a simultaneous solution of $J_{\phi}(u, 1)=0$ and of $K_{\phi}(u, 1)=0$. If $\Delta_{\phi} \neq 0$, and $r_{1}(\varphi) \neq r_{2}(\varphi) \neq r_{3}(\varphi), r_{1}(\varphi) \neq r_{3}(\varphi), \varphi$ will be said to be general, otherwise special. Clearly if $\varphi$ is general $r_{1}, r_{2}$, and $r_{3}$ are all free. 
An iterative field is a set of functions of $n$ variables which is closed with respect to the following operations:

(1) permutation of any two arguments;

(2) substitution of two identical arguments for two different arguments;

(3) substitution of a function of the set for any argument.

Two operations are said to be iteratively equivalent if any iterative field containing either contains the other.

An iterative characteristic of an operation is a property which belongs to all operations iteratively equivalent to that operation.

\section{Theorems.}

I. The free roots of $L_{\phi}(u, 1)=0$ constitute iterative characteristics of $\varphi$.

For $L_{\phi}(u, 1)=0$ is equivalent to $\varphi(u, u)=u$, unless $u$ is a simultaneous root of $J_{\phi}(u, 1)=0$ and of $K_{\phi}(u, 1)=0$. Now, $\varphi(u, u)=u$ is a property of $\varphi$ invariant under iteration. If $r$ is a root of $L_{\phi}(u, 1)=0$ that is not free, then in general $\varphi(r, r)=r$ is not significant and determinate. If this latter equation is significant, let us subject our number system to a linear transformation that changes $r$ to $\infty$. It may readily be seen that two operations will retain their iterative relationships unchanged under such transformations, that roots of $L_{\phi}(u, 1)=0$ will remain roots, and that free roots will remain free. $\varphi$ will then become a $\psi$ of the form $A+B x+C y$. This operation can only be iteratively equivalent to operations of its own sort, for all of which $\infty$ is a root that is not free. This completes the proof of our theorem. As a corollary we obtain

II. No general bilinear operation is equivalent to any special bilinear operation.

III. If $\varphi$ and $\psi$ are reduced equivalent general bilinear operations, then

$$
\begin{aligned}
A_{\phi} & =A_{\psi}, \\
B_{\phi}+C_{\phi}-E_{\phi} & =B_{\psi}+C_{\psi}-E_{\psi}, \\
F_{\phi}+G_{\phi}-D_{\phi} & =F_{\psi}+G_{\psi}-D_{\psi}, \\
H_{\phi} & =H_{\psi} .
\end{aligned}
$$

Lemma 1. If $\varphi$ is reduced, $u$ and $v$ can be chosen relatively prime in such a manner that $J_{\phi}(u, v)$ is prime to $K_{\phi}(u, v)$. 
Proof. Let $N$ be the H.C.F. of $A_{\phi}, B_{\phi}+C_{\phi}$, and $D_{\phi}$. Let $M$ be the product of all prime factors in $\Delta_{\phi}$ but not in $N$. Let $P$ be the product of all the primes in $M$, but not in $A_{\phi}$ nor in $D_{\phi}$. Let $Q$ be the product of all primes in $M$ and $A_{\phi}$, but not in $D_{\phi}$. Let $R$ be the product of all primes in $M$ and $D_{\phi}$, but not in $A_{\phi}$. Let $S$ be the product of all primes in $N$, but neither in $E_{\phi}$ nor in $H_{\phi}$. Let $T$ be the product of all primes in $N$ and $E_{\phi}$, but not in $H_{\phi}$. Let $U$ be the product of all primes in $N$ and $H_{\phi}$, but not in $E_{\phi}$. Let $u=P R S U$, $v=Q T$. We get as a result that $J_{\phi}$ is prime to $M$, while $K_{\phi}$ is prime to $N$. Now, it may readily be shown that any factor common to $J_{\phi}$ and $K_{\phi}$ belongs to $\Delta_{\phi}$. Since, however, any prime factor of $\Delta_{\phi}$ belongs either to $M$ or to $N$, it is either prime to $J_{\phi}$ or to $K_{\phi} *$

Lemma 2. If $a$ and $b$ are any two numbers not both containing $a$ prime $p$ and if $c$ and $d, e$ and $f$, are couples of the same sort, while then

$$
a d-b c \equiv 0\left(\bmod p^{k}\right), \quad a f-b e \equiv 0\left(\bmod p^{k}\right),
$$

$$
c f-d e \equiv 0\left(\bmod p^{k}\right) .
$$

This lemma, whose proof is immediate, enables us to classify fractions into sets modulo $p^{k}$. We shall say that

if

$$
a / b \equiv c / d\left(\bmod p^{k}\right)
$$

$$
a d-b c \equiv 0\left(\bmod p^{k}\right)
$$

while $a$ and $b$ do not contain $p$ in common, nor do $c$ and $d$.

Lemma 3. If $u / v$ and $w / z$ are in their lowest terms, and

$$
u / v \equiv w / z\left(\bmod p^{k}\right),
$$

then if $\varphi$ is any bilinear operation such that

it follows that

$$
\varphi(u / v, u / v) \equiv u / v\left(\bmod p^{k}\right),
$$

$$
\varphi(w / z, w / z) \equiv w / z\left(\bmod p^{k}\right) .
$$

The proof of lemma 3 offers no difficulty.

Combining lemmas 2 and 3 , it is at once clear that the equation

$$
\varphi(u / v, u / v) \equiv u / v\left(\bmod p^{k}\right)
$$

for a given $u / v$ and $p^{k}$ is an iterative characteristic of $\varphi$.

* Cf. Mathews, Theory of Numbers, vol. 1, p. 132. 
We are now in a position to take up the proof of Theorem III. Clearly $\varphi(u / v, u / v)$ is equal to $J_{\phi}(u, v) / K_{\phi}(u, v)$. Let $u$ and $v$ be two numbers such that $J_{\phi}(u, v)$ and $K_{\phi}(u, v)$ are prime to one another. By lemma 1, such numbers exist. Then to say that

$$
J_{\phi}(u, v) / K_{\phi}(u, v) \equiv u / v\left(\bmod p^{k}\right)
$$

is equivalent to saying

$$
L_{\phi}(u, v) \equiv 0\left(\bmod p^{k}\right),
$$

or that $p^{k}$ is a factor of $L_{\phi}(u, v)$. If $\varphi$ is iteratively equivalent to $\psi$, clearly we must have

$$
L_{\psi}(u, v) \equiv 0\left(\bmod p^{k}\right) .
$$

That is, every prime power that divides $L_{\phi}$ must also divide $L_{\psi}$, or in other words, $L_{\phi}$ is a factor of $L_{\psi}$ for a particular set of values of $u$ and $v$. However, since by Theorem I the equations $L_{\phi}(u, 1)=0$ and $L_{\psi}(u, 1)=0$ have the same three distinct roots, $L_{\psi}$ is a constant multiple of $L_{\phi}$. Hence $L_{\phi}$ is always a factor of $L_{\psi}$. Likewise, $L_{\psi}$ is always a factor of $L_{\phi}$. Hence, apart from a possible difference of sign, $L_{\phi}$ and $L_{\psi}$ are identical. This proves Theorem III.

Massachusetts Institute of Technology, April 16, 1920.

\section{NECESSARY AND SUFFICIENT CONDITIONS THAT A LINEAR TRANSFORMATION BE COMPLETELY CONTINUOUS.}

BY PROFESSOR CHARLES ALBERT FISCHER.

(Read before the American Mathematical Society December 31, 1919.)

A LARGE part of the Fredholm theory of integral equations has been derived for the equation

$$
h(x)=f(x)+\lambda T(f),
$$

where $T(f)$ is a completely continuous linear transformation.* It has also been proved that every linear transformation, that

* F. Riesz, Acta Mathematica, vol. 41 (1918), pp. 71-98. 